MATHEMATICS OF COMPUTATION

Volume 73, Number 248, Pages 1827-1847

S 0025-5718(04)01667-9

Article electronically published on May 11, 2004

\title{
SPECTRAL DIVISION METHODS FOR BLOCK GENERALIZED SCHUR DECOMPOSITIONS
}

\author{
XIAOBAI SUN AND ENRIQUE S. QUINTANA-ORTÍ
}

\begin{abstract}
We provide a different perspective of the spectral division methods for block generalized Schur decompositions of matrix pairs. The new approach exposes more algebraic structures of the successive matrix pairs in the spectral division iterations and reveals some potential computational difficulties. We present modified algorithms to reduce the arithmetic cost by nearly $50 \%$, remove inconsistency in spectral subspace extraction from different sides (left and right), and improve the accuracy of subspaces. In application problems that only require a single-sided deflating subspace, our algorithms can be used to obtain a posteriori estimates on the backward accuracy of the computed subspaces with little extra cost.
\end{abstract}

\section{INTRODUCTION}

In this paper we provide a different perspective of the so-called spectral division methods and present modified algorithms for computing block generalized Schur decompositions [12, 25] of a matrix pair $(A, B)$, where $A$ and $B$ are square matrices of order $n$. The problem we are concerned with is to efficiently construct two unitary matrices $Q=\left(Q_{1}, Q_{2}\right)$ and $Z=\left(Z_{1}, Z_{2}\right)$ so that

$$
\begin{aligned}
& Q^{\mathrm{H}} A Z=\left(\begin{array}{c}
Q_{1}^{\mathrm{H}} \\
Q_{2}^{\mathrm{H}}
\end{array}\right) A\left(Z_{1}, Z_{2}\right)=\left(\begin{array}{cc}
A_{11} & A_{12} \\
0 & A_{22}
\end{array}\right), \\
& Q^{\mathrm{H}} B Z=\left(\begin{array}{c}
Q_{1}^{\mathrm{H}} \\
Q_{2}^{\mathrm{H}}
\end{array}\right) B\left(Z_{1}, Z_{2}\right)=\left(\begin{array}{cc}
B_{11} & B_{12} \\
0 & B_{22}
\end{array}\right),
\end{aligned}
$$

and $\mathcal{L}_{1}=\lambda\left(A_{11}, B_{11}\right)$, the set of the generalized eigenvalues of the matrix pencil $A_{11}-\lambda B_{11}$, is disjoint from $\mathcal{L}_{2}=\lambda\left(A_{22}, B_{22}\right)=\lambda(A, B)-\lambda\left(A_{11}, B_{11}\right)$. Notice that $Z_{1}$ in (1.1) is an orthonormal basis for the right-deflating subspace corresponding to $\mathcal{L}_{1}$. A subspace $\mathcal{X}$ is a right-deflating subspace of $(A, B)$ if $\operatorname{dim}(A \mathcal{X}+B \mathcal{X}) \leq$ $\operatorname{dim}(\mathcal{X})$, where the equality holds if $(A, B)$ is regular [12]. A left-deflating subspace of $(A, B)$ is a right-deflating subspace of $\left(A^{\mathrm{H}}, B^{\mathrm{H}}\right)$. Hereafter, we assume that $(A, B)$ is regular, and we shorten generalized eigenvalues to eigenvalues where the meaning is clear from the context.

By recursively applying the decomposition in (1.1), partial or complete information of the eigenspectrum of $(A, B)$ can be obtained. In many applications deflating subspaces with respect to a specified division of the spectrum are also of

Received by the editor August 13, 2002.

2000 Mathematics Subject Classification. Primary 65F15; Secondary 15A18, 15A22.

Key words and phrases. Generalized eigenproblem, matrix sign and disc functions, spectral divide-and-conquer algorithms. 
interest (in analysis and synthesis of linear control systems, e.g., optimal control problems, Kalman filtering, model reduction, stabilization of descriptor systems, etc. [1, 5, 6, 17, [27]).

A spectral division method for the decomposition (1.1) consists of two main steps. In the first step, a spectral projector is computed that divides the spectrum by mapping two complementary subsets of $\lambda(A, B), \mathcal{L}_{1}$ and $\mathcal{L}_{2}$, to two distinct values and preserves the respective spectral subspaces. Well-known examples of spectral projectors are obtained from the matrix sign function and the matrix disc function [22]. Procedures for computing these matrix functions are essentially iterative. Once the spectral projector is available, the second step is to extract an orthonormal basis for a spectral subspace. The subspace extraction algorithm in general varies depending on the type of matrix function available.

Let $Z_{1}$ be an orthonormal basis for the right-deflating subspace corresponding to $\mathcal{L}_{1}$ and let $Q_{2}$ be an orthonormal basis of the left-deflating subspace corresponding to $\mathcal{L}_{2}$. Then, mathematically, $Q_{2}^{\mathrm{H}} A Z_{1}=Q_{2}^{\mathrm{H}} B Z_{1}=0$, and the matrix pair $(A, B)$ is decoupled into two smaller ones, as shown in (1.1). Computationally, $\left(Q_{2}^{\mathrm{H}} A Z_{1}, Q_{2}^{\mathrm{H}} B Z_{1}\right)$ indicates how accurate the decoupling of matrix pair $(A, B)$ is. Also, given a pair of unitary matrices $Q=\left(Q_{1}, Q_{2}\right)$ and $Z=\left(Z_{1}, Z_{2}\right)$, $\left(Q_{2}^{\mathrm{H}} A Z_{1}, Q_{2}^{\mathrm{H}} B Z_{1}\right)$ can be used to obtain normwise bounds on the backward perturbation $(E, F)$ in $(A+E, B+F)$ corresponding to the decoupled matrix pair

$$
\left(\begin{array}{cc}
Q_{1}^{\mathrm{H}}(A+E) Z_{1} & Q_{1}^{\mathrm{H}}(A+E) Z_{2} \\
0 & Q_{2}^{\mathrm{H}}(A+E) Z_{2}
\end{array}\right), \quad\left(\begin{array}{cc}
Q_{1}^{\mathrm{H}}(B+F) Z_{1} & Q_{1}^{\mathrm{H}}(B+F) Z_{2} \\
0 & Q_{2}^{\mathrm{H}}(B+F) Z_{2}
\end{array}\right) .
$$

When only a deflating subspace (e.g., $Z_{1}$ ) is desired, if $Q_{2}$ can be obtained with little extra cost, the computed $\left(Q_{2}^{\mathrm{H}} A Z_{1}, Q_{2}^{\mathrm{H}} B Z_{1}\right)$ can be used to estimate the backward accuracy of the computed orthonormal bases.

The use of the matrix sign function as a spectral division tool was first introduced by Roberts 22] for the standard eigenvalue problem (i.e., the case in which $B=I$, the identity matrix). Consider that matrix $A$ has no eigenvalues on the imaginary axis and denote by

$$
A=S\left(\begin{array}{cc}
J^{-} & 0 \\
0 & J^{+}
\end{array}\right) S^{-1}
$$

its Jordan decomposition, with $J^{-}$of size $k \times k$ and $J^{+}$of size $(n-k) \times(n-k)$ containing the Jordan blocks corresponding to the eigenvalues in the open left complex plane, $\mathbb{C}^{-}$, and open right complex plane, $\mathbb{C}^{+}$, respectively. The matrix sign function of $A$ is then defined as

$$
\operatorname{sign}(A) \stackrel{\text { def }}{=} S\left(\begin{array}{cc}
-I_{k} & 0 \\
0 & I_{n-k}
\end{array}\right) S^{-1} .
$$

Assuming $A$ is nonsingular, one procedure for computing $\operatorname{sign}(A)$ is to use the Newton iteration for the equation $X^{2}-I=0$ with $A$ as the initial iterate. This procedure was extended by Gardiner and Laub [10] for the generalized eigenvalue problem; see Figure 1 The iteration converges if $(A, B)$ has no eigenvalues on the imaginary axis, and the asymptotic convergence rate is quadratic. Each iteration invokes one matrix inverse and two matrix multiplications. The inverse, however, may be sensitive to perturbations. There are inverse-free iteration schemes for the matrix sign function based on polynomial approximations to the inverse [14]. Such schemes, however, have bounded convergence regions. 


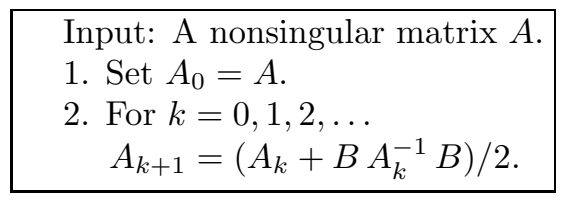

Figure 1. The Newton iteration for the matrix sign function.

Assume the sequence of $A_{k}$ generated by the Newton iteration for the matrix sign function converges to $A_{\infty}$. Matrix $\left(B-A_{\infty}\right) / 2$ is the spectral projector corresponding to the eigenvalues of $(A, B)$ in $\mathbb{C}^{-}$. Given a spectral projector, many effective rank-revealing algorithms developed in recent years can be used for the basis extraction; see [7, 9, 13, 21, 20].

The matrix disc function was also first defined by Roberts in 22 for the standard eigenvalue problem. Assume $A$ has no eigenvalues on the unit circle and rewrite its Jordan decomposition as

$$
A=T\left(\begin{array}{cc}
J^{0} & 0 \\
0 & J^{\infty}
\end{array}\right) T^{-1}
$$

with $J^{0}$ of size $l \times l$ and $J^{\infty}$ of size $(n-l) \times(n-l)$ containing the Jordan blocks corresponding to the eigenvalues inside and outside the unit circle, respectively. The matrix disc function of $A$ is then defined [5] as

$$
\operatorname{disc}(A) \stackrel{\text { def }}{=} T\left(\begin{array}{cc}
I_{l} & 0 \\
0 & 0
\end{array}\right) T^{-1}
$$

Malyshev presents a scheme based on the matrix disc function [18] for the spectral division of matrix pairs. "Malyshev's iteration", in Figure 2, is first applied to the matrix pair. The theoretical foundation of this iteration can be traced to works of Godunov [11], Bulgakov and Godunov [8], Kublanovskaya [15, 16], and others (see the references therein). Each step of Malyshev's iteration uses a QR factorization and two matrix multiplications. The sequence of $\left(A_{k}+B_{k}\right)^{-1} B_{k}$ in Malyshev's iteration converges if $(A, B)$ has no eigenvalues on the unit circle; see [18] and [3]. Under the same condition, the asymptotic convergence rate is also quadratic. On the convergence of Malyshev's iteration, $\left(A_{\infty}+B_{\infty}\right)^{-1} B_{\infty}$ is the spectral projector corresponding to the eigenvalues of $(A, B)$ inside the unit circle.

The spectral division algorithm proposed by Malyshev was modified by Bai, Demmel, and $\mathrm{Gu}$ [3] to make it completely inverse-free. First, they present an

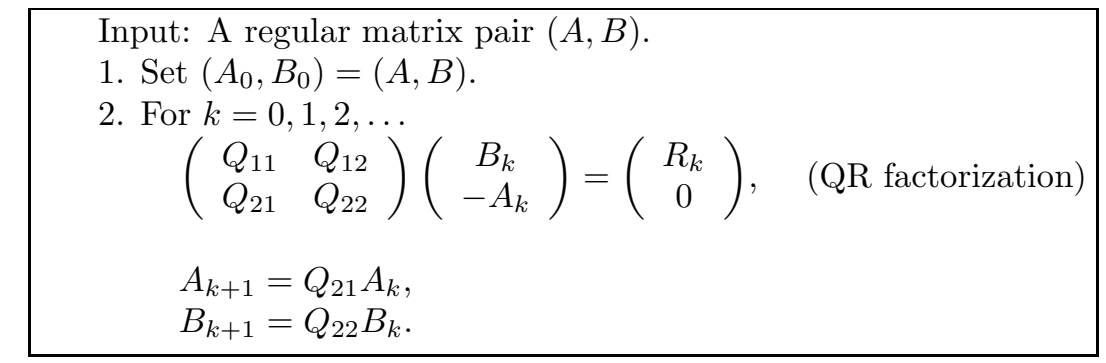

Figure 2. Malyshev's iteration for the matrix disc function. 
effective stopping criterion for Malyshev's iteration based on the Cholesky factors of the QR factorizations. Secondly, the bases for the deflating subspaces are obtained by means of a generalized QR factorization [3], without explicitly constructing $\left(A_{\infty}+B_{\infty}\right)^{-1} B_{\infty}$

The above spectral division methods are attractive in that (1) the computation of spectral subspaces, unlike the QZ algorithm [19, does not depend on the computation of the eigenvalues and the reordering of the computed eigenvalues; and (2) the algorithmic building blocks are mainly triangular factorizations (LR factorizations or QR factorizations) and matrix multiplications. High-performance codes for such matrix computations are available on a broad class of high-performance computers and on parallel computers in particular [2, 4, 21].

The analysis that we present in this paper exposes more algebraic structures of the successive matrix pairs in the spectral division (Newton and Malyshev's) iterations and reveals some potential computational difficulties in the subspace extraction stage. Let $(A, B)$ be a matrix pair without eigenvalues on the imaginary axis. Then, $A$ is nonsingular and $(A, B)$ is regular. We have the following results for the spectral division algorithms based on the matrix sign function: (1) if $B$ is nonsingular, the sequence of $A_{k}$ converges to a matrix, $A_{\infty}$, that can be considered the generalized matrix sign function of $A$ associated with $B$; $(2)$ the matrix pair $\left(A_{\infty}, B\right)$ is regular and preserves both the left- and right-deflating subspaces of $(A, B)$ corresponding to the eigenvalues in $\mathbb{C}^{-}$and $\mathbb{C}^{+}$, respectively; (3) if $B$ is singular, the iteration may still converge, at a linear rate, and the subspace extraction on both sides (left and right) is inconsistent; and (4) the singularity or near-singularity of $B$ leads to the singularity or near-singularity of the limit matrix $A_{\infty}$ and causes inconsistency in subspace extraction from both sides.

Malyshev's iteration admits a matrix pair $(A, B)$ with $A$ and/or $B$ singular, without breakdown. We prove, without assuming the nonsingularity of either $A$ or $B$, that the sequence of $\left(A_{k}, B_{k}\right)$ converges to a regular matrix pair if and only if $(A, B)$ has no eigenvalues on the unit circle, which implies that $(A, B)$ is regular and that $\left(A_{k}, B_{k}\right)$ may still converge if there is an eigenvalue on the unit circle. Although a step-by-step mapping relationship exists between the Newton iteration and Malyshev's iteration (see [3, 5]), Malyshev's iteration preserves only the rightdeflating subspaces. To decouple a matrix pair into two smaller ones, Bai, Demmel, and $\mathrm{Gu}[3]$ propose to repeat Malyshev's iteration with $\left(A_{0}, B_{0}\right)=\left(A^{\mathrm{H}}, B^{\mathrm{H}}\right)$ and then to obtain the left-deflating subspace from the converged matrix pair. Two such independent iterations (one for each subspace, left and right) may lead to numerically inconsistent decoupling, in addition to the doubled arithmetic cost. The extra cost is too high if the left-deflating subspace is only needed to obtain error estimates.

We present a subspace extraction technique which provides bases for both the left- and right-deflating subspaces with a single Malyshev iteration on $(A, B)$ (thus we avoid repetition of Malyshev's iteration on $\left.\left(A^{\mathrm{H}}, B^{\mathrm{H}}\right)\right)$. The arithmetic cost of our one-sided spectral division algorithm is reduced by nearly $50 \%$ in comparison to the methods based on two-sided spectral division algorithms [3]. The deflation scheme also reduces the decoupling inconsistency caused by independent iterations and/or independent subspace extractions on both sides.

Our paper is organized as follows. In Section 2, we review two canonical forms of matrix pencils. In Section 3 we expose the algebraic structure of the sequence 
of a matrix pair with the Newton iteration and present our deflation scheme for the generalized Schur decomposition (1.1). In Section 4 we provide an analysis of Malyshev-like algorithms and present a scheme for accurate extraction of left- and right-deflating subspaces which only requires an iteration. In Section 5 we describe initial transformations that allow the spectrum to be divided in various ways. In Section [6] we present some numerical experiments. Our concluding remarks are in Section 7

We use the following notation and conventions in addition to what has been introduced so far. The matrix norm $\|\cdot\|$ denotes the 2-norm and $\kappa(X)$ denotes the condition number of $X$ with respect to inversion. The tensor product (or right Kronecker product) of an $m \times m$ matrix $A$ and an $n \times n$ matrix $B$ is defined as the $m n \times m n$ matrix

$$
A \otimes B=\left(\begin{array}{ccc}
a_{11} B & \ldots & a_{1 m} B \\
\vdots & \ddots & \vdots \\
a_{m 1} B & \ldots & a_{m m} B
\end{array}\right) .
$$

We denote by $\mathcal{R}(X)$ and $\mathcal{N}(X)$ the range and the null space of matrix $X$, respectively. In some cases it is necessary to write the generalized eigenvalue problem in the symmetrical cross-product form, as suggested in [24]. If $(\alpha, \beta) \neq 0$ and $\operatorname{det}(\beta A-\alpha B)=0$, then $(\alpha, \beta)$ represents an eigenvalue of $(A, B)$ in the sense that, for any $\delta \neq 0$, we have $\operatorname{det}(\delta \beta A-\delta \alpha B)=0$ also. The corresponding eigenvalueeigenvector problem is

$$
\beta A x=\alpha B x, \quad(\alpha, \beta) \neq 0, x \neq 0 .
$$

In other cases we use for convenience the traditional, asymmetrical forms $A x=\lambda B x$ or $\mu A x=B x$. When $\beta \neq 0$, we simply set $\beta=1$ and hence $\alpha=\lambda$. When $\beta=0$, we set $\alpha=1$. We denote by $\rho(A)$ the spectral radius of a square matrix $A$.

\section{BLOCK CANONICAL DECOMPOSITIONS}

The following two theorems from [25] on the two canonical forms for matrix pencils underpin the unified theory we present for the spectral division algorithms.

\subsection{Generalized Schur form.}

Theorem 2.1. Let $(A, B)$ be a regular matrix pair. Then there are unitary matrices $Q$ and $Z$ such that $S=Q^{\mathrm{H}} A Z$ and $T=Q^{\mathrm{H}} B Z$ are upper triangular with the diagonal element pairs $\left(s_{i i}, t_{i i}\right), i=1, \ldots, n$, being the eigenvalues of $(A, B)$ in a prespecified order.

Proof. The proof is by induction on the order $n$. The theorem holds for $n=1$. Assume that the theorem holds true for all matrix pairs of order less than $n, n>1$. Let $z_{1},\left\|z_{1}\right\|_{2}=1$, be an eigenvector corresponding to the first eigenvalue pair $(\alpha, \beta)$, namely,

$$
\beta A z_{1}=\alpha B z_{1}, \quad(\beta, \alpha) \neq 0 .
$$

Then, the rank of $\left(A z_{1}, B z_{1}\right)$ is no greater than 1 . By the regularity of the matrix pair, $\left(A z_{1}, B z_{1}\right) \neq 0$. There is a vector $q_{1},\left\|q_{1}\right\|_{2}=1$, such that $\left(A z_{1}, B z_{1}\right)=$ $q_{1}\left(s_{11}, t_{11}\right)=q_{1}(\delta \alpha, \delta \beta)$ for some scalar $\delta \neq 0$. Let $Z_{c}=\left(z_{1}, Z_{2}\right)$ and $Q_{r}=\left(q_{1}, Q_{2}\right)$ be unitary matrices. Then,

$$
Q_{r}^{\mathrm{H}} A Z_{c}=\left(\begin{array}{cc}
s_{11} & S_{12} \\
0 & A_{n-1}
\end{array}\right), \quad Q_{r}^{\mathrm{H}} B Z_{c}=\left(\begin{array}{cc}
t_{11} & T_{12} \\
0 & B_{n-1}
\end{array}\right),
$$


and $\lambda(A, B)=\left\{\left(s_{11}, t_{11}\right)\right\} \cup \lambda\left(A_{n-1}, B_{n-1}\right)$. Notice that $\left(A_{n-1}, B_{n-1}\right)$ is also regular. By the induction hypothesis, there are unitary matrices $Z_{n-1}$ and $Q_{n-1}$ such that $Q_{n-1}^{\mathrm{H}} A_{n-1} Z_{n-1}$ and $Q_{n-1}^{\mathrm{H}} B_{n-1} Z_{n-1}$ are triangular with their diagonal elements in a specified order. The induction step is completed by letting $Q=Q_{r} \operatorname{diag}\left(1, Q_{n-1}\right)$ and $Z=Z_{c} \operatorname{diag}\left(1, Z_{n-1}\right)$.

The above proof can be extended to the following cases:

(a) The Schur decomposition for the standard eigenvalue problem. We can always let $Q_{r}=Z_{c}$ in the induction step and have, by induction, $Q_{n}=Z_{n}$.

(b) The generalized real Schur decomposition for a real, regular matrix pair $(A, B)$. The matrices $T$ and $S$ are block triangular matrices with $1 \times 1$ and $2 \times 2$ matrix pairs on the diagonals.

(c) The generalized Schur decomposition for a singular matrix pair. See 26].

We are interested in the case in which $(A, B)$ has more than one distinctive eigenvalue. Let $\mathcal{L}_{1}$ and $\mathcal{L}_{2}$ be two nonempty subsets of $\lambda(A, B)$. They are called complementary subsets of $\lambda(A, B)$ if $\mathcal{L}_{1} \cup \mathcal{L}_{2}=\lambda(A, B)$ and $\mathcal{L}_{1} \cap \mathcal{L}_{2}=\emptyset$.

Corollary 2.2. Let $(A, B)$ be a regular pair. Let $\mathcal{L}_{1}$ and $\mathcal{L}_{2}$ be a pair of complementary subsets of $\lambda(A, B)$. Then there are unitary matrices $Q$ and $Z$ such that

$$
Q^{\mathrm{H}} A Z=\left(\begin{array}{cc}
A_{11} & A_{12} \\
0 & A_{22}
\end{array}\right), \quad Q^{\mathrm{H}} B Z=\left(\begin{array}{cc}
B_{11} & B_{12} \\
0 & B_{22}
\end{array}\right), \quad \begin{aligned}
& \lambda\left(A_{11}, B_{11}\right)=\mathcal{L}_{1}, \\
& \lambda\left(A_{22}, B_{22}\right)=\mathcal{L}_{2} .
\end{aligned}
$$

\subsection{Block diagonal forms.}

Theorem 2.3. Let $(A, B)$ be a regular matrix pair. Let $\mathcal{L}_{1}$ and $\mathcal{L}_{2}$ be a pair of complementary subsets of $\lambda(A, B)$. Then there are nonsingular matrices $X$ and $Y$ such that

$$
Y^{-1} A X=\left(\begin{array}{cc}
A_{11} & 0 \\
0 & A_{22}
\end{array}\right), \quad Y^{-1} B X=\left(\begin{array}{cc}
B_{11} & 0 \\
0 & B_{22}
\end{array}\right), \quad \begin{aligned}
& \lambda\left(A_{11}, B_{11}\right)=\mathcal{L}_{1}, \\
& \lambda\left(A_{22}, B_{22}\right)=\mathcal{L}_{2} .
\end{aligned}
$$

Theorem 2.3 can be applied recursively to one or two of the decoupled matrix pairs $\left(A_{11}, B_{11}\right)$ and $\left(A_{22}, B_{22}\right)$, when needed. In the $2 \times 2$ block diagonal canonical form, let $W^{\mathrm{H}}=Y^{-1}$ and partition $X=\left(X_{1}, X_{2}\right)$ and $W^{\mathrm{H}}=\left(\begin{array}{c}W_{1}^{\mathrm{H}} \\ W_{2}^{\mathrm{H}}\end{array}\right)$ in conformity with the block diagonal matrix pair. Then, for $i=1,2, X_{i}$ and $W_{i}^{\mathrm{H}}$ represent, respectively, the right- and left-deflating subspaces of $(A, B)$ corresponding to $\mathcal{L}_{i}$. The diagonal form renders more relationships between the deflating subspaces. In particular, with the same assumptions as in Theorem 2.3. we have the following corollaries.

Corollary 2.4. The right- and left-deflating subspaces of $(A, B)$ corresponding to $\mathcal{L}_{1}$ and $\mathcal{L}_{2}$, respectively, are complementary.

Let $\mathcal{X}$ be a deflating subspace of $(A, B)$. We call the subspace $\mathcal{Y}=A \mathcal{X}+B \mathcal{X}$ the image of $\mathcal{X}$ under $(A, B)$. In (2.1), $\mathcal{R}\left(Y_{1}\right)$ is the image of $\mathcal{R}\left(X_{1}\right)$. For simplicity, we say that $Y_{1}$ is the image of $X_{1}$ under $(A, B)$.

Corollary 2.5. The left-deflating subspace of $(A, B)$ corresponding to $\mathcal{L}_{2}\left(\mathcal{L}_{1}\right)$ is orthogonally complementary to the image of the right-deflating subspace corresponding to $\mathcal{L}_{1}\left(\mathcal{L}_{2}\right.$.) 


\section{Spectral Division VIA THE MATRIX Sign FUNCtion}

In this section we provide an analysis on the convergence of the Newton iteration (see Figure 1), and we describe the algebraic structure of the converged matrix, including the case in which matrix $B$ is singular. The newly revealed algebraic structure suggests that a single Newton iteration is sufficient to obtain the deflating subspaces on both sides. Moreover, we provide a deflation scheme to eliminate numerical inconsistency in subspace extraction.

3.1. The Newton iteration for the matrix sign function. The Newton iteration requires that $A_{0}(=A)$ be nonsingular. Let $A_{k} x=\lambda^{(k)} B x, k \geq 0$, where $x \neq 0$ is an eigenvector of $\left(A_{k}, B\right)$ corresponding to the eigenvalue $\lambda^{(k)}, 0<\left|\lambda^{(k)}\right|<\infty$. Assume for the moment that $A_{k}$ is nonsingular. Then,

$$
A_{k+1} x=\frac{1}{2}\left(\lambda^{(k)}+\frac{1}{\lambda^{(k)}}\right) B x .
$$

We see that, at iteration $k, \lambda^{(k)}$ is mapped to the eigenvalue $\left(\lambda^{(k)}+\lambda^{(k)^{-1}}\right) / 2$ of $\left(A_{k+1}, B\right)$; the eigenvector is preserved. Similarly, the left eigenvector is preserved as well. We will show that the eigenvector-preserving property of the Newton iteration can be extended to spectral subspaces. The iteration

$$
\lambda^{(k+1)}=\left(\lambda^{(k)}+1 / \lambda^{(k)}\right) / 2, \quad k \geq 0,
$$

converges if and only if $\lambda^{(0)}$ is not on the imaginary axis, that is, $\operatorname{Re}\left(\lambda^{(0)}\right) \neq 0$. For $\lambda^{(0)}$ with $\operatorname{Re}\left(\lambda^{(0)}\right) \neq 0, \lim _{k \rightarrow \infty} \lambda^{(k)}=\operatorname{sign}\left(\lambda^{(0)}\right)$. We assume for the rest of this section that $(A, B)$ has no eigenvalues on the imaginary axis in the $\lambda$-form, thereby implying that $A$ is nonsingular.

Although the inverse of $B$ is not required implicitly in the iteration, the singularity or near-singularity of $B$ causes the singularity or near-singularity of $A_{k}$, even when $A_{0}=A$ is well conditioned. To see this, we consider the case in which $B$ is singular and its zero eigenvalues are all simple. By Theorem [2.3] there are nonsingular matrices $X=\left(X_{0}, X_{1}, X_{2}\right)$ and $Y=\left(Y_{0}, Y_{1}, Y_{2}\right)$ such that

$$
A_{0} X=Y\left(\begin{array}{ccc}
A_{00}^{(0)} & 0 & 0 \\
0 & A_{11}^{(0)} & 0 \\
0 & 0 & A_{22}^{(0)}
\end{array}\right), \quad B X=Y\left(\begin{array}{ccc}
0 & 0 & 0 \\
0 & B_{11} & 0 \\
0 & 0 & B_{22}
\end{array}\right) \text {, }
$$

where $B_{11}$ and $B_{22}$ are nonsingular, $\lambda\left(A_{11}^{(0)}, B_{11}\right) \subset \mathbb{C}^{-}$, and $\lambda\left(A_{22}^{(0)}, B_{22}\right) \subset \mathbb{C}^{+}$. It can be verified directly that

$$
A_{k+1} X=Y\left(\begin{array}{ccc}
A_{00}^{(k)} / 2 & 0 & 0 \\
0 & \left(A_{11}^{(k)}+B_{11}\left(A_{11}^{(k)}\right)^{-1} B_{11}\right) / 2 & 0 \\
0 & 0 & \left(A_{22}^{(k)}+B_{22}\left(A_{22}^{(k)}\right)^{-1} B_{22}\right) / 2
\end{array}\right) .
$$

Thus, $A_{k+1}$ is nonsingular if $A_{k}$ is nonsingular. As $k \rightarrow \infty, A_{11}^{(k)} \rightarrow-B_{11}, A_{22}^{(k)} \rightarrow$ $B_{22}$, but $A_{00}^{(k)} \rightarrow 0$. In other words, the sequence of $A_{k}$ converges to a singular matrix $A_{\infty}$,

$$
A_{\infty}=Y\left(\begin{array}{ccc}
0 & 0 & 0 \\
0 & -B_{11} & 0 \\
0 & 0 & B_{22}
\end{array}\right) X^{-1}
$$


Generally, it can be proved that if the Jordan blocks with eigenvalues at 0 are of order less than 3 , then the $A_{k}$ converge. Notice that in the case in which $B$ is singular and the $A_{k}$ converge, the convergence rate is linear, and the limit matrix pair $\left(A_{\infty}, B\right)$ is singular.

The above analysis also includes the case in which $B$ is nonsingular, that is, $A_{00}^{(0)}$ is empty. In such a case we have shown that the Newton iteration converges if and only if $(A, B)$ has no imaginary eigenvalue in the $\lambda$-form. Moreover, the asymptotic convergence rate is quadratic, and the limit matrix pair $\left(A_{\infty}, B\right)$ is regular. Let $A$ and $B$ be nonsingular, and let $(A, B)$ be a matrix pair without imaginary eigenvalues in the $\lambda$-form. We define the generalized matrix sign function of $(A, B)$ as the following:

$$
\operatorname{sign}(A, B) \stackrel{\text { def }}{=} Y\left(\begin{array}{cc}
-B_{11} & 0 \\
0 & B_{22}
\end{array}\right) X^{-1}
$$

The function is well defined in that it is independent of the selection of bases for the spectral subspaces. When $B=I, \operatorname{sign}(A, B)=\operatorname{sign}(A)$. Notice that the roles of $A$ and $B$ in $\operatorname{sign}(A, B)$ are not symmetrical in that if $B_{11}$ or $B_{22}$ is near-singular, so is $\operatorname{sign}(A, B)$. We have proved the following theorem.

Theorem 3.1. Let $(A, B)$ be a matrix pair without eigenvalues on the imaginary axis. Then, the sequence of $\left(A_{k}, B\right)$ by the Newton iteration converges to a regular matrix pair if and only if $B$ is nonsingular. Moreover, $\lim _{k \rightarrow \infty} A_{k}=\operatorname{sign}(A, B)$.

3.2. Subspace extraction and deflation algorithms. A deflation algorithm for the Schur decomposition (1.1) requires $Z_{1}$, an orthonormal basis for the rightdeflating subspace corresponding to the eigenvalues, for instance, in $\mathbb{C}^{-}$, and $Q_{2}$, an orthonormal basis for the left-deflating subspace corresponding to the eigenvalues in $\mathbb{C}^{+}$.

There are two approaches for extracting the spectral subspaces. One approach is to form the spectral projectors first. The projectors can be expressed in terms of $\operatorname{sign}(A, B)$ and $B$ when $B$ is nonsingular and $(A, B)$ has no eigenvalues on the imaginary axis:

$$
P_{X_{1}}=X \operatorname{diag}(I, 0) X^{-1}=B^{-1}(B-\operatorname{sign}(A, B)) / 2, \quad P_{X_{2}}=I-P_{X_{1}} .
$$

Given a projector, many effective rank-revealing algorithms developed in recent years can be used to obtain an orthonormal basis for the subspace; see [7, 9, 13. 2120 . To avoid the inverse of $B$, practitioners use the rank-revealing algorithms to obtain a basis for a spectral subspace without forming the projector. The alternative approach is based on the following direct relationships between the limit matrix pair $\left(A_{\infty}, B\right)$ and the spectral subspaces:

$$
\mathcal{N}(B+\operatorname{sign}(A, B))=\mathcal{R}\left(X_{1}\right), \quad \mathcal{N}(B-\operatorname{sign}(A, B))=\mathcal{R}\left(X_{2}\right) .
$$

Our analysis in Section 3.1 shows that $\left(A_{\infty}, B\right)$ preserves the left-deflating subspaces as well. Recall that $W=Y^{-\mathrm{H}}=\left(W_{1}, W_{2}\right)$ and $W_{1}^{\mathrm{H}}$ and $W_{2}^{\mathrm{H}}$ are the left-deflating subspaces. Specifically, we have

$$
P_{W_{1}}=W \operatorname{diag}(I, 0) W^{-1}=B^{-\mathrm{H}}(B-\operatorname{sign}(A, B))^{\mathrm{H}} / 2, \quad P_{W_{2}}=I-P_{W_{1}},
$$

and

$$
\mathcal{N}\left(B^{\mathrm{H}}+\operatorname{sign}(A, B)^{\mathrm{H}}\right)=\mathcal{R}\left(W_{1}\right), \quad \mathcal{N}\left(B^{\mathrm{H}}-\operatorname{sign}(A, B)^{\mathrm{H}}\right)=\mathcal{R}\left(W_{2}\right) .
$$


In other words, we can obtain the deflating subspace on both sides from $A_{\infty}$ and $B$.

We now show that the independent subspace extraction on both sides may cause numerical inconsistency in subspace extraction. To see this, we consider the case in which $B$ is singular and the $A_{k}$ converge to a singular matrix $A_{\infty}$ as shown in (3.1). We have

$$
\begin{aligned}
& \mathcal{N}\left(B+A_{\infty}\right)=\mathcal{R}\left(\left[X_{0}, X_{1}\right]\right), \quad \mathcal{N}\left(B-A_{\infty}\right)=\mathcal{R}\left(\left[X_{0}, X_{2}\right]\right), \\
& \mathcal{N}\left(B^{\mathrm{H}}+A_{\infty}^{\mathrm{H}}\right)=\mathcal{R}\left(\left[W_{0}, W_{1}\right]\right), \quad \mathcal{N}\left(B^{\mathrm{H}}-A_{\infty}^{\mathrm{H}}\right)=\mathcal{R}\left(\left[W_{0}, W_{2}\right]\right) .
\end{aligned}
$$

The extraction of the spectral subspaces on one side is not consistent with that on the other side. The subspaces $\mathcal{N}\left(B-A_{\infty}\right)$ and $\mathcal{N}\left(B^{\mathrm{H}}+A_{\infty}^{\mathrm{H}}\right)$ are no longer orthogonal. Such inconsistency is also expected in numerical computation when $B$ is near-singular. Our approach to circumventing the problem is based on Corollary [2.5. Specifically, we use the fact that $\mathcal{R}\left(W_{2}\right)$ is orthogonally complementary to the image of $\mathcal{R}\left(\left[X_{0}, X_{1}\right]\right)$ under $(A, B)$, namely,

$$
\begin{aligned}
& W_{2}^{\mathrm{H}}\left(A\left[X_{0}, X_{1}\right], B\left[X_{0}, X_{1}\right]\right)=0, \\
& \operatorname{dim}\left(\left(A\left[X_{0}, X_{1}\right], B\left[X_{0}, X_{1}\right]\right)\right)+\operatorname{dim}\left(W_{2}\right)=n .
\end{aligned}
$$

We call this approach the Schur deflation approach. It can be applied if the Newton iteration converges. Let $Z_{1}$ and $Q_{2}$ be orthonormal bases for $\mathcal{R}\left(\left[X_{0}, X_{1}\right]\right)$ and $\mathcal{R}\left(W_{2}\right)$, respectively. The procedure for computing $Z_{1}$ and $Q_{2}$ can be composed of two rank-revealing QR (RRQR) factorizations; see Figure 3. By a proof similar to that of Theorem 2.1. one can see that the use of an RRQR procedure, instead of a QR factorization procedure, is necessary in the Schur deflation step.

We note that the Schur deflation technique was first used by Van Dooren [26] for computing reducing subspaces of singular pencils; the use of the technique in the spectral division methods for the block Schur decomposition (1.1) and for the error estimate is new to the best knowledge of the authors.

Given $(A, B)$. Assume $A_{\infty}$ is obtained by the Newton iteration in Figure 1.

1. Construct a unitary matrix $\bar{Z}=\left(Z_{1}, Z_{2}\right)$, using an RRQR procedure, such that

$$
\Pi_{Q}\left(B+A_{\infty}\right)\left(Z_{1}, Z_{2}\right)=\left(\begin{array}{cc}
0 & L_{12} \\
0 & L_{22}
\end{array}\right),
$$

where $\Pi_{Q}$ can be a permutation matrix.

2. The Schur deflation. Construct a unitary matrix $Q=\left(Q_{1}, Q_{2}\right)$, using an RRQR procedure, such that

$$
Q^{\mathrm{H}}\left(A Z_{1}, B Z_{1}\right) \Pi_{Z}=\left(\begin{array}{cc}
S_{11} & T_{11} \\
0 & 0
\end{array}\right),
$$

where $\Pi_{Z}$ can be a permutation matrix.

The matrix pair $\left(Q^{\mathrm{H}} A Z, Q^{\mathrm{H}} B Z\right)$ is decoupled as in (1.1).

Figure 3. Subspace extraction and the Schur decomposition via the matrix sign function. 


\section{Spectral Division VIA the MATRiX DisC FUnCtion}

In this section we provide an analysis on the convergence of Malyshev's iteration (see Figure 2), and we describe the algebraic structure of the converged matrix pair, including the case in which there are eigenvalues on the unit circle. Although Malyshev's iteration does not preserve the left-deflating subspaces, the Schur deflation scheme introduced in Section 3 still applies. The new difficulty lies in subspace extraction due to the element decrease in Malyshev's iteration. We provide a modified algorithm for subspace extraction.

4.1. Malyshev's iteration for the matrix disc function. We first introduce Malyshev-like iterations with Malyshev's iteration as a special instance and then provide the convergence conditions for Malyshev's iteration. Given $\left(A_{0}, B_{0}\right)$, a Malyshev-like iteration generates two matrix pencil sequences $\left(A_{k}, B_{k}\right)$ and $\left(C_{k}, S_{k}\right), k=1,2, \ldots$, by the following two rules.

The CS-AB EQUivalenCE RULE.

$$
S_{k} A_{k}=C_{k} B_{k}, \quad \text { where } \operatorname{rank}\left(\left[C_{k}, S_{k}\right]\right)=n .
$$

THE SQUARING RECURRENCE.

$$
\begin{aligned}
& A_{k+1}=C_{k} A_{k}, \\
& B_{k+1}=S_{k} B_{k} .
\end{aligned}
$$

We may also call the second rule the cross-update rule. Malyshev's iteration uses the QR factorization of $\left(B_{k}^{\mathrm{H}},-A_{k}^{\mathrm{H}}\right)^{\mathrm{H}}$ to generate $C_{k}$ and $S_{k}$. It imposes the orthonormal condition on $\left(C_{k}, S_{k}\right)$ in the CS-AB equivalence rule.

The matrix pair $\left(C_{k}, S_{k}\right)$ is equivalent to $\left(A_{k}, B_{k}\right)$ if the latter is regular. By the regularity of $\left(A_{k}, B_{k}\right)$, there are $\mu_{1}$ and $\mu_{2}$ such that $\mu_{1} A_{k}+\mu_{2} B_{k}$ is nonsingular. Let $\beta A_{k} x=\alpha B_{k} x$, where $x \neq 0$ and $(\alpha, \beta) \neq 0$. Then, we have by the CS-AB equivalence rule (4.1),

$$
\beta C_{k}\left(\mu_{1} A_{k}+\mu_{2} B_{k}\right) x=\alpha \mu_{1} C_{k} B_{k} x+\beta \mu_{2} S_{k} A_{k} x=\alpha S_{k}\left(\mu_{1} A_{k}+\mu_{2} S_{k} B_{k}\right) x .
$$

We see that $\lambda\left(A_{k}, B_{k}\right)=\lambda\left(C_{k}, S_{k}\right)$ and the right eigenvectors of $\left(C_{k}, S_{k}\right)$ are those of $\left(A_{k}, B_{k}\right)$ transformed by $\mu_{1} A_{k}+\mu_{2} B_{k}$. Since $\left(C_{k}, S_{k}\right)$ is also regular, the left equivalence transformation between the two matrix pairs can be represented by a linear combination of $C_{k}$ and $S_{k}$. We can therefore expect a squaring of the eigenvalues of $\left(A_{k}, B_{k}\right)$ in $\left(A_{k+1}, B_{k+1}\right)$ formed by the cross-update rule (4.2). Indeed, we have

$$
\beta^{2} A_{k+1} x=\beta^{2} C A_{k} x=\alpha \beta C B_{k} x=\alpha \beta S A_{k} x=\alpha^{2} S B_{k} x=\alpha^{2} B_{k+1} x .
$$

Assume that $\beta A_{0} x=\alpha B_{0} x$, where $x \neq 0$ and $(\alpha, \beta) \neq 0$. Then, by induction on $k$,

$$
\beta^{2^{k}} A_{k} x=\alpha^{2^{k}} B_{k} x, \quad k \geq 0 .
$$

By the convention introduced in Section 1 we set $\beta=1$ when $|\alpha|<|\beta|(\lambda=\alpha / \beta$ is inside the unit circle in $\mathbb{C}$ ), and we set $\alpha=1$ otherwise. As $k \rightarrow \infty$,

$$
\begin{array}{llll}
\left(\alpha^{2^{k}}, 1\right) \rightarrow(0,1), & |\alpha|<1 ; & \left(1, \beta^{2^{k}}\right)=(1,1), & \beta=1 ; \\
\left(1, \beta^{2^{k}}\right) \rightarrow(1,0), & |\beta|<1 ; & \left(1, \beta^{2^{k}}\right) \text { do not converge, } & |\beta|=1, \beta \neq 1 .
\end{array}
$$

The following example shows that the divergence of an eigenvalue sequence $\left(1, \beta^{2^{k}}\right),|\beta|=1, \beta \neq 1$, does not necessarily imply the divergence of the matrix pair sequence $\left(A_{k}, B_{k}\right)$. 
Example 4.1. Let $B_{0}=F$ be a nonsingular matrix and let $A_{0}=\left(\begin{array}{cc}e^{i \theta} & 0 \\ 0 & 0\end{array}\right) F$ for a real number $\theta$. Then, $(A, B)$ is a regular pair and has an eigenvalue on the unit circle. Let $S_{0}=\left(\begin{array}{cc}e^{i \theta} / \sqrt{2} & 0 \\ 0 & 1\end{array}\right)$ and $C_{0}=\left(\begin{array}{cc}1 / \sqrt{2} & 0 \\ 0 & 0\end{array}\right)$. Then, $\left(S_{0}, C_{0}\right)$ satisfies the CS-AB equivalence rule (4.1) and the orthogonality condition in Malyshev's iteration. Form $\left(A_{1}, B_{1}\right)$ by the cross-update rule (4.2), that is, $A_{1}=C_{0} A_{0}$ and $B_{1}=S_{0} B_{0}$. For all $k \geq 0$, we have

$$
\begin{array}{rlrl}
A_{k} & =\left(\begin{array}{cc}
e^{i 2^{k} \theta}(1 / \sqrt{2})^{k} & 0 \\
0 & 0
\end{array}\right) F, & B_{k} & =\left(\begin{array}{cc}
(1 / \sqrt{2})^{k} & 0 \\
0 & 1
\end{array}\right) F, \\
C_{k}=\left(\begin{array}{cc}
1 / \sqrt{2} & 0 \\
0 & 0
\end{array}\right), & & S_{k}=\left(\begin{array}{cc}
e^{i 2^{k} \theta} / \sqrt{2} & 0 \\
0 & 1
\end{array}\right) .
\end{array}
$$

The matrix pair at convergence is singular.

In Malyshev's iteration the sequence of $\left\|R_{k}\right\|$ is monotonically nonincreasing and always converges. However, Malyshev's iteration at each step is not deterministic in that $U\left(C_{k}, S_{k}\right)$, for an arbitrary unitary matrix $U$, also satisfies the first rule and the orthonormal condition. Therefore, we say that the sequence of $\left(A_{k}, B_{k}\right)$ is convergent up to a unitary matrix in the sense that there is a sequence of unitary matrices $U_{k}$ such that the sequence of $\left(U_{k} A_{k}, U_{k} B_{k}\right)$ converges. Assume that $\left(A_{0}, B_{0}\right)$ has eigenvalues inside and outside the unit circle. Then, we have shown the following.

(a) If the sequence of $\left(A_{k}, B_{k}\right)$ converges, up to a unitary factor, to a matrix pencil $\left(A_{\infty}, B_{\infty}\right)$, then both $A_{\infty}$ and $B_{\infty}$ are singular.

(b) If either $C_{k}$ or $S_{k}$ is kept unitary, the sequence of $\left(A_{k}, B_{k}\right)$ is not convergent.

(c) The condition that $\left(A_{0}, B_{0}\right)$ has no eigenvalues on the unit circle is not a necessary condition for Malyshev's iteration to converge.

Example 4.1 also shows that for the case in which there is an eigenvalue on the unit circle, $\left(A_{k}, B_{k}\right)$ converge, the convergence rate is linear, and the limit matrix pair is singular. For the case in which there are no eigenvalues on the unit circle, we have the following theorem.

Theorem 4.2. If $\left(A_{0}, B_{0}\right)$ has no eigenvalues on the unit circle, then the sequence of $\left(A_{k}, B_{k}\right)$ by Malyshev's iteration (Figure 2) converges to a regular matrix pair, up to a unitary factor. Moreover, the sequence of $R_{k}$ converges asymptotically quadratically.

Proof. We may assume without loss of generality that $\left(A_{0}, B_{0}\right)$ has eigenvalues both inside and outside the unit circle. By Theorem 2.3, there are two nonsingular matrices $X$ and $Y$ such that

$$
A_{0} X=Y\left(\begin{array}{cc}
I & 0 \\
0 & A_{22}
\end{array}\right), \quad B_{0} X=Y\left(\begin{array}{cc}
B_{11} & 0 \\
0 & I
\end{array}\right)
$$

where $\rho\left(B_{11}\right)<1, \rho\left(A_{22}\right)<1$. For a Malyshev-like iteration, we have

$$
S_{0}=G \operatorname{diag}\left(B_{11}, I\right) Y^{-1}, \quad C_{0}=G \operatorname{diag}\left(I, A_{22}\right) Y^{-1},
$$

for some nonsingular matrix $G$, and

$$
\left(C_{0} A_{0}\right) X=G \operatorname{diag}\left(I, A_{22}^{2}\right), \quad\left(S_{0} B_{0}\right) X=G \operatorname{diag}\left(B_{11}^{2}, I\right) .
$$


For Malyshev's iteration, $G=U L^{-1}$, where $U$ is a unitary matrix and $L$ is the Cholesky factor of the matrix $\left(\operatorname{diag}\left(B_{11}, I\right) Y^{-1}, \operatorname{diag}\left(I, A_{22}\right) Y^{-1}\right)$.

Let $G_{0}=Y$, and let $G_{0}=U_{0} L_{0}^{-1}$ be a QL factorization of $G_{0}$. We have that, for $L_{k}$ nonsingular,

$$
A_{k} X=G_{k}\left(\begin{array}{cc}
I & 0 \\
0 & A_{22}^{2^{k}}
\end{array}\right), \quad B_{k} X=G_{k}\left(\begin{array}{cc}
B_{11}^{2^{k}} & 0 \\
0 & I
\end{array}\right), \quad k \geq 0
$$

where

$$
G_{k+1}=U_{k+1} L_{k+1}^{-1}, \quad k \geq 0,
$$

for some unitary matrix $U_{k+1}$, and $L_{k+1}$ is the Cholesky factor of

$$
\left(\operatorname{diag}\left(B_{11}^{2^{k}}, I\right) L_{k}, \operatorname{diag}\left(I, A_{22}^{2^{k}}\right) L_{k}\right) .
$$

Let $M^{(k)}=L_{k} L_{k}^{\mathrm{H}}$ and partition $M^{(k)}$ in conformity with the block diagonal matrices. Then,

$$
\begin{aligned}
M^{(k+1)} & =\left(\begin{array}{cc}
I & 0 \\
0 & A_{22}^{2^{k}}
\end{array}\right) M^{(k)}\left(\begin{array}{cc}
I & 0 \\
0 & A_{22}^{2^{k}}
\end{array}\right)^{\mathrm{H}}+\left(\begin{array}{cc}
B_{11}^{2^{k}} & 0 \\
0 & I
\end{array}\right) M^{(k)}\left(\begin{array}{cc}
B_{11}^{2^{k}} & 0 \\
0 & I
\end{array}\right)^{\mathrm{H}} \\
& =\left(\begin{array}{cc}
M_{11}^{(k)}+B_{11}^{2^{k}} M_{11}^{(k)}\left(B_{11}^{2^{k}}\right)^{\mathrm{H}} & B_{11}^{2^{k}} M_{12}^{(k)}+M_{12}^{(k)}\left(A_{22}^{2^{k}}\right)^{\mathrm{H}} \\
M_{21}^{(k)}\left(B_{11}^{2^{k}}\right)^{\mathrm{H}}+A_{22}^{2^{k}} M_{21}^{(k)} & M_{22}^{(k)}+A_{22}^{2^{k}} M_{22}^{(k)}\left(A_{22}^{2^{k}}\right)^{\mathrm{H}}
\end{array}\right) .
\end{aligned}
$$

Let $\rho_{1}=\rho\left(B_{11}\right), \rho_{2}=\rho\left(A_{22}\right)$ and $\rho=\max \left(\rho_{1}, \rho_{2}\right)$. Then, $\rho<1$. As $k \rightarrow \infty$,

$$
\begin{aligned}
& \left\|M_{21}^{(k+1)}\right\|=\left\|M_{12}^{(k+1)}\right\| \leq 2 \rho^{2^{k}}\left\|M_{12}^{(k)}\right\| \leq 2^{k+1} \rho^{\sum_{j=0}^{k} 2^{j}}\left\|M_{12}^{(0)}\right\| \rightarrow 0, \\
& \left\|M_{i i}^{(k)}\right\| \leq\left\|M_{i i}^{(k+1)}\right\| \leq\left(1+\rho_{i}^{2^{k+1}}\right)\left\|M_{i i}^{(k)}\right\|<e^{\gamma_{i}}\left\|M_{i i}^{(0)}\right\|, \quad i=1,2, \\
& \inf \left(M_{i i}^{(k+1)}\right) \geq \inf \left(M_{i i}^{(k)}\right) \geq \inf \left(M_{i i}^{(0)}\right), \quad i=1,2,
\end{aligned}
$$

where

$$
\gamma_{i}=\rho_{i}^{2} \frac{1-2 \ln \left(\rho_{i}\right)}{12 \ln \left(\rho_{i}\right)^{2}}, \quad i=1,2
$$

Recall that for $i=1,2$, the values $M_{i i}^{(0)}$ are equal to $\left(Y^{-1} Y^{-\mathrm{H}}\right)_{i i}$; hence they are nonsingular. We have shown that the $M^{(k)}$ converge, as $k \rightarrow \infty$, to a nonsingular block diagonal matrix $M_{\infty}$. Let $L_{\infty}$ be the Cholesky factor of $M_{\infty}$. Then, $L_{\infty}$ is also block diagonal, $L_{\infty}=\operatorname{diag}\left(L_{11}, L_{22}\right)$. By (4.4) and (4.5), $\left(A_{k}, B_{k}\right)$ converge to a regular matrix pair $\left(A_{\infty}, B_{\infty}\right)$, up to a unitary factor,

$$
A_{\infty}=U\left(\begin{array}{cc}
L_{11}^{-1} & 0 \\
0 & 0
\end{array}\right) X^{-1}, \quad B_{\infty}=U\left(\begin{array}{cc}
0 & 0 \\
0 & L_{22}^{-1}
\end{array}\right) X^{-1},
$$

where $U$ is a unitary matrix. Let $R_{k}$ be the Cholesky factor of $\left(A_{k}^{\mathrm{H}}, B_{k}^{\mathrm{H}}\right)^{\mathrm{H}}$. Then, for any nonzero vector $v$,

$$
v^{\mathrm{H}} X^{-\mathrm{H}} \operatorname{diag}\left(M_{11}^{(k)}, M_{22}^{(k)}\right)^{-1} X^{-1} v \leq v^{\mathrm{H}}\left(R_{k}^{\mathrm{H}} R_{k}\right) v \leq 2 v^{\mathrm{H}} X^{-\mathrm{H}}\left(M^{(k)}\right)^{-1} X^{-1} v,
$$

and, as $k \rightarrow \infty$,

$$
R_{k}^{\mathrm{H}} R_{k} \rightarrow R_{\infty}^{\mathrm{H}} R_{\infty}=X^{-\mathrm{H}} M^{-1} X^{-1} .
$$

By (4.6), the asymptotic convergence rate of $R_{k}$ is quadratic. 


$$
\left\|R_{k}-R_{k-1}\right\|_{1} \leq 10 n \epsilon\left\|R_{k}\right\|_{1} . \epsilon: \text { the machine precision }
$$

FIGURE 4. The BDG stopping criterion.

We see that while the convergence of $\left(A_{k}, B_{k}\right)$ is up to a unitary factor, the Cholesky factors $R_{k}$ converge and are readily available to be used in convergence criteria. Bai, Demmel, and Gu use this convergence to design the "BDG" stopping criterion shown in Figure 4

4.2. Subspace extraction and deflation algorithms. From Theorem 4.2 we have the following corollary for extracting bases of the right-deflating subspaces.

Corollary 4.3. Assume $\left(A_{0}, B_{0}\right)$ has no eigenvalues on the unit circle. Let $\mathcal{X}_{1}$ and $\mathcal{X}_{2}$ be the right-deflating subspaces corresponding to the eigenvalues inside and outside the unit circle, respectively. Then, for Malyshev's iteration, $A_{k}+B_{k}, k \geq 0$, are nonsingular, and

(1) $\lim _{k \rightarrow \infty}\left(A_{k}+B_{k}\right)^{-1} A_{k} \rightarrow\left(A_{\infty}+B_{\infty}\right)^{-1} A_{\infty}=P_{X_{1}}$, the projector for $\mathcal{X}_{1}$, and

(2) $\mathcal{N}\left(B_{\infty}\right)=\mathcal{X}_{1}, \quad \mathcal{N}\left(A_{\infty}\right)=\mathcal{X}_{2}$

The projector $P_{X_{1}}$ is the matrix disc function of $(A+B)^{-1} A$ [5]. We call $\left(A_{\infty}, B_{\infty}\right)$ in (4.7) the generalized matrix disc function of $\left(A_{0}, B_{0}\right)$. The computation of the projectors requires the inverse of $A_{\infty}+B_{\infty}$, which may be close to a singular matrix. Part (2) of Corollary 4.3 suggests an alternative way, which can also be extended to the case in which $\left(A_{0}, B_{0}\right)$ has eigenvalue(s) on the unit circle and $\left(A_{k}, B_{k}\right)$ converge. In this case, $\left(A_{\infty}+B_{\infty}\right)$ is singular, and $\mathcal{N}\left(B_{\infty}\right)$ and $\mathcal{N}\left(A_{\infty}\right)$ are not complementary. More specifically, $\mathcal{N}\left(B_{\infty}\right) \cap \mathcal{N}\left(A_{\infty}\right)$ is the right-deflating subspace corresponding to the eigenvalues on the unit circle. We study the case with eigenvalue(s) on the unit circle in order to gain insights into

Given $(A, B)$. Assume $\left(A_{\infty}, B_{\infty}\right)$ is obtained by Malyshev's iteration in Figure 2,

1. Construct a unitary matrix $\bar{Z}=\left(Z_{1}, Z_{2}\right)$, using an RRQR procedure, such that

$$
\Pi_{Q} B_{\infty}\left(Z_{1}, Z_{2}\right)=\left(\begin{array}{cc}
0 & L_{11} \\
0 & L_{22}
\end{array}\right),
$$

where $\Pi_{Q}$ can be a permutation matrix.

2. The Schur deflation. Construct a unitary matrix $Q=\left(Q_{1}, Q_{2}\right)$, using an RRQR procedure, such that

$$
Q^{\mathrm{H}}\left(A Z_{1}, B Z_{1}\right) \Pi_{Z}=\left(\begin{array}{cc}
S_{11} & T_{11} \\
0 & 0
\end{array}\right),
$$

where $\Pi_{Z}$ can be a permutation matrix.

The matrix pair $\left(Q^{\mathrm{H}} A Z, Q^{\mathrm{H}} B Z\right)$ is decoupled as in (1.1).

Figure 5. Subspace extraction and the Schur decomposition via the matrix disc function. 


$$
\begin{aligned}
& \text { 1.1. }\left(\begin{array}{ll}
A_{\infty} & B_{\infty}
\end{array}\right)=R\left(\begin{array}{ll}
U_{A} & U_{B}
\end{array} \quad\right. \text { ( RQ factorization ). } \\
& \text { 1.2. } U_{A} Z=\left(\begin{array}{cc}
0 & L_{12} \\
0 & L_{22}
\end{array}\right) \quad(\text { RR-RQF }) .
\end{aligned}
$$

Figure 6. Modification of step 1.

the case with eigenvalue(s) near the unit circle. The deflation algorithm in Figure 5 is based on part (2) of Corollary 4.3 and on Corollary 2.5. It is inverse-free and requires only one-sided Malyshev iteration. To obtain the right-deflating subspace of $(A, B)$ corresponding to the eigenvalues outside and on the unit circle, if any, we replace $B_{\infty}$ by $A_{\infty}$ in Step 1.

Recall that $\left\|R_{k}\right\|$ is nonincreasing. Since $\left\|A_{\infty}\right\|,\left\|B_{\infty}\right\| \leq\left\|R_{\infty}\right\|$, the numerical computation for revealing the null space of $A_{\infty}$ or $B_{\infty}$ by step 1 of the algorithm in Figure 5 may be difficult. By the convergence analysis in Section 4.1 the numerical rank of $A_{\infty}$, or $B_{\infty}$, can be significantly affected by $\kappa\left(L_{11}\right)$ and $\kappa(X)$. From the proof of Theorem 4.2 we know that $\kappa\left(L_{11}\right)$ is related to $\kappa(Y)$. We modify step 1 using the scheme in Figure 6 In step 1.1, if the Cholesky factor $R$ is nonsingular, then $\mathcal{N}\left(A_{\infty}\right)$ and $\mathcal{N}\left(U_{A}\right)$ are complementary, and the singular values of $U_{A}$ (or $\left.U_{B}\right)$ are at around 1 and 0 , according to the theory of the CS decomposition [12. In other words, step 1.1 is the preconditioning stage for the next rank-revealing step. Similarly, the scheme can be applied to obtain the right-deflating subspace corresponding to the eigenvalues outside the unit circle (and on the unit circle, if any).

\section{TRANSFormations OF SPECTRAL DiVISIONS}

The spectrum of a matrix pair can be divided in various ways by using a spectral division algorithm, as discussed in the preceding sections, combined with an initial spectral transformation. The Möbius transformations of a given matrix pair $(A, B)$ are particularly simple, involving only linear combinations of $A$ and $B$. We have the following theorem from [25].

Theorem 5.1. Let $M$ be a nonsingular $2 \times 2$ matrix. Given the matrix pair $(A, B)$, set $C=A\left(M \otimes I_{n}\right)$ and $D=B\left(M \otimes I_{n}\right)$. Given a pair $(\alpha, \beta) \neq(0,0)$ such that $\operatorname{det}(\beta A-\alpha B) \neq 0$, define $(\gamma, \delta)$ by

$$
\left(\begin{array}{c}
\delta \\
-\gamma
\end{array}\right)=M^{-1}\left(\begin{array}{c}
\beta \\
-\alpha
\end{array}\right)
$$

Then $\operatorname{det}(\beta A-\alpha B) \neq 0$ if and only if $\operatorname{det}(\delta C-\gamma D) \neq 0$.

In the preceding theorem, if we choose for example to use Malyshev's iteration, then the Cayley transformation on $(A, B)$

$$
M=\left(\begin{array}{cc}
1 & 1 \\
1 & -1
\end{array}\right), \quad\left(A_{0}, B_{0}\right)=(A+B, A-B),
$$

enables the division of $\lambda(A, B)$ along the imaginary axis. Figures 7 and 8 depict the progressive changes of the eigenvalues with Malyshev's iteration starting, respectively, from $(A+B, A-B)$ and $(A, B)$, where $A$ and $B$ are the matrices in Example 2 of Section 6. Each figure consists of four snapshots, showing the distribution of the initial eigenvalues (top left) and the eigenvalues at the end of iteration 1 (top right), iteration 2 (bottom left), and iteration 5 (bottom right). 

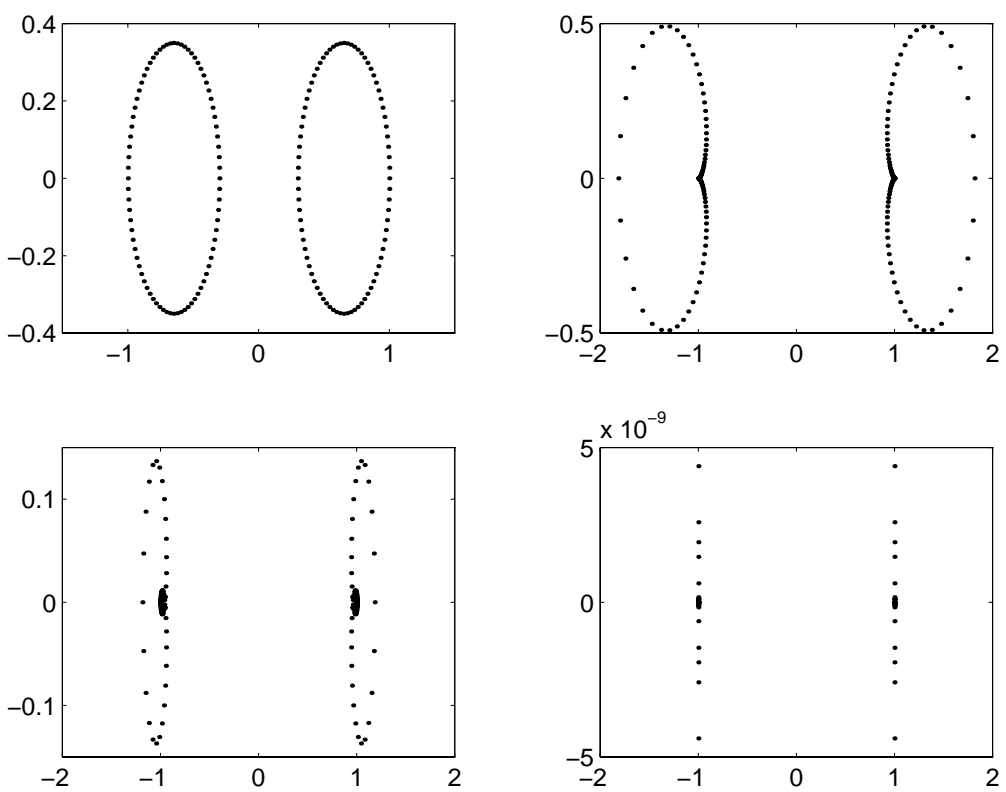

FIgURE 7. Iterations for the matrix sign function.
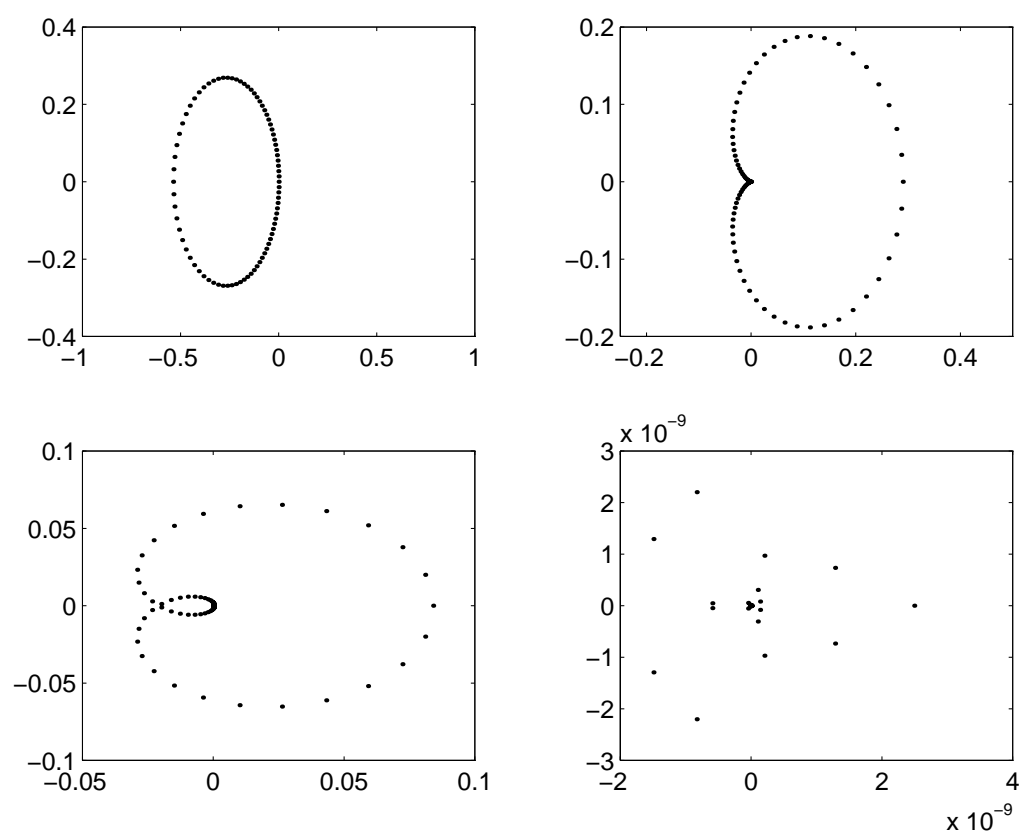

FiguRE 8. Iterations for the matrix disc function. 
In Figure 7, the eigenvalues converge to their sign function values \pm 1 ; in Figure 8 we only show the eigenvalues inside the unit circle, which converge to 0 . The reciprocal of the eigenvalues outside the circle is symmetric to those inside with respect to the imaginary axis (see Section 4). Notice that the scale of the axis is different for each figure.

Among other Möbius transformations there are those scaling the unit circle, shifting the circle center, and shifting and/or rotating the imaginary axis. In [3], different spectral transformations are used at different decoupling levels to locate the eigenvalues in a specified region in the complex plane. These transformations were first employed as acceleration techniques in some fine-grained algorithms, such as the $\mathrm{AB}$ algorithms [16, for generalized eigenvalue problems.

\section{NumERICAL EXPERIMENTS}

In this section we present some numerical experiments with spectral division algorithms via the matrix disc function. Specifically, we compare the following algorithms:

MM-INV: The spectral division algorithm originally designed by Malyshev [18]. Malyshev's iteration is applied twice, starting with matrix pairs $(A, B)$ and $\left(A^{\mathrm{H}}, B^{\mathrm{H}}\right)$. The corresponding spectral projectors, $P_{X_{1}}$ and $P_{W_{2}}$, are explicitly constructed and the left- and right-deflating subspaces are then obtained from those via an RRQR factorization.

MM-GQR: The spectral division algorithm proposed by Bai, Demmel and Gu [3]. Malyshev's iteration is also applied twice, on $(A, B)$ and $\left(A^{\mathrm{H}}, B^{\mathrm{H}}\right)$, but now the left- and right-deflating subspaces are obtained by means of two generalized QR factorizations [3, without explicit construction of the spectral projectors.

M-ABQR: Our new spectral division algorithm. Malyshev's iteration is only applied once, on $(A, B)$. The subspace extraction scheme described in Figure 5 is then employed to obtain the left- and right-deflating subspaces.

The three algorithms use the BDG stopping criterion (see Figure 4) in Malyshev's iteration (Figure 2). They differ in the iterations performed (one-sided in our algorithm and two-sided in algorithms MM-INV and MM-GQR) and the subspace extraction technique. Our spectral division algorithm only requires one iteration on a matrix pair and therefore approximately $50 \%$ of the computational cost of algorithms MM-INV and MM-GQR.

To measure and compare the backward accuracy of these algorithms, we evaluate the relative decoupling residual $(r d r)$ :

$$
\mathrm{rdr}=\left\|\left(E_{21}, F_{21}\right)\right\|_{F} /\|(A, B)\|_{F} .
$$

All the experiments are performed in MATLAB 4.2 on a Sun SPARCstation-20 using IEEE arithmetic with the machine precision $\epsilon \approx 2.2 \times 10^{-16}$.

In all our experiments the three algorithms take about the same number of iterations to compute the right-deflating subspace for each matrix pair. With twosided iterations, the number of right iterations and the number of left iterations are very close. 
We conduct two sets of experiments. The matrices in the first set are $100 \times 100$ random matrices with entries independent and normally distributed within $[-1,1]$. We apply the algorithms for both the division along the imaginary axis and the division along the unit circle (see Section 5). The decoupling residuals with MMGQR and M-ABQR are of about the same order for both division cases, whereas the decoupling residuals by MM-INV are more sensitive to $\kappa\left(A_{\infty}+B_{\infty}\right)$.

The second set of experiments consists of matrix pairs from $[1,3]$. The problems are designed with known eigenstructures which can be adjusted to obtain difficult cases for the spectral division algorithms. We present experimental results for three examples with the algorithms using the spectrum division along the imaginary axis. In these examples, $B=I$. We use the matrix pair $(A, I)$ and the equivalent pair $\left(R, Q^{\mathrm{H}}\right)$, where $A=Q R$ is a $\mathrm{QR}$ factorization. The two options show no noticeable differences. Matlab files for these examples are available with PRISM project working note \#32 at http://www.mcs.anl.gov/prism.

Following the notation in [3], we use $\Delta(A)$ to denote the gap between the eigenvalues and the imaginary axis:

$$
\Delta(A)=\min _{\lambda_{j} \in \lambda(A)}\left|\operatorname{Re}\left(\lambda_{j}\right)\right| .
$$

The larger the gap $\Delta(A)$ is, the faster $\left(A_{k}, B_{k}\right)$ converge. To estimate the sensitivity of the spectral subspaces to perturbations in a matrix pair, we compute a lower bound of the quantity dif introduced in [23]:

$$
\stackrel{\operatorname{dif}\left[\left(A_{11}, B_{11}\right),\left(A_{22}, B_{22}\right)\right]}{\stackrel{\text { def }}{=} \inf _{\max \left\{\|P\|_{\mathrm{F}},\|Q\|_{\mathrm{F}}\right\}=1} \max \left\{\left\|Q A_{11}+A_{22} P\right\|_{\mathrm{F}},\left\|Q B_{11}+B_{22} P\right\|_{\mathrm{F}}\right\} .}
$$

The lower bound we compute is the smallest singular value of the matrix operator $T$ that maps $(Q, P)$ to $\left(Q A_{11}+A_{22} P, Q B_{11}+B_{22} P\right)$ (see [23]).

Example 1. The matrix $A$ is real and Hamiltonian:

$$
A=Q^{\mathrm{T}}\left(\begin{array}{rr}
F & S \\
G & -F^{\mathrm{T}}
\end{array}\right) Q,
$$

where

$$
F=\left(\begin{array}{rrrr}
-\eta & 1 & 0 & 0 \\
-1 & -\eta & 0 & 0 \\
0 & 0 & \eta & 1 \\
0 & 0 & -1 & \eta
\end{array}\right), \quad S=G=\left(\begin{array}{l}
1 \\
1 \\
1 \\
1
\end{array}\right)\left(\begin{array}{llll}
1 & 1 & 1 & 1
\end{array}\right)
$$

and $Q$ is an orthogonal matrix obtained from the $\mathrm{QR}$ factorization of a random matrix. The eigenvalues of $A$ are symmetric with respect to the imaginary axis as well as the real axis and are in a neighborhood of $\pm \eta^{2} \pm i$. This type of matrix arises in an optimal control problem [1, 17. Table 1 lists the values of dif, iteration number, and relative decoupling residual with a few different values of $\eta$. As $\eta \rightarrow 0$, the eigenvalues at about $\pm \eta^{2} \pm i$ approach two points $\pm i$ on the imaginary axis, and the division along this axis becomes more difficult. All three algorithms behave about the same numerically; however, algorithm M-ABQR only requires a one-sided iteration and therefore uses only about $50 \%$ of the arithmetic operations used by the other two algorithms. 
TABLE 1. Experimental results with Example 1.

\begin{tabular}{|c|c|c|c|c|c|}
\hline $\begin{array}{c}\Delta(A) \approx \\
\eta^{2} / 2\end{array}$ & dif & iter & $\begin{array}{c}\text { MM-INV } \\
\text { rdr }\end{array}$ & $\begin{array}{c}\text { MM-GQR } \\
\text { rdr }\end{array}$ & $\begin{array}{c}\text { M-ABQR } \\
\text { rdr }\end{array}$ \\
\hline $5.00 e-1$ & $4.26 e-1$ & 8 & $2.04 e-16$ & $2.31 e-16$ & $1.81 e-16$ \\
\hline $5.00 e-3$ & $5.04 e-3$ & 15 & $4.66 e-15$ & $4.75 e-15$ & $6.52 e-15$ \\
\hline $5.00 e-5$ & $5.00 e-5$ & 22 & $3.84 e-13$ & $3.84 e-13$ & $2.55 e-13$ \\
\hline $5.00 e-7$ & $5.00 e-7$ & 28 & $3.91 e-11$ & $3.91 e-11$ & $1.53 e-11$ \\
\hline
\end{tabular}

Example 2. The matrix is of Hamiltonian-like structure:

$$
A=Q^{\mathrm{T}}\left(\begin{array}{cc}
A_{11} & A_{12} \\
0 & A_{22}
\end{array}\right) Q
$$

with

$$
A_{11}=\left(\begin{array}{cccc}
1-\alpha & & & \alpha \\
\alpha & 1-\alpha & & \\
& \ddots & \ddots & \\
& & \alpha & 1-\alpha
\end{array}\right), \quad A_{22}=-A_{11}^{\mathrm{T}},
$$

where $A_{12}$ is a $k \times k$ random matrix (not necessarily symmetric), $Q$ is an orthogonal matrix, and $A_{11}$ is a circulant matrix with parameter $\alpha$. The eigenvalues of $A_{11}$ are evenly distributed on a circle with center at $1-\alpha$ and radius $\alpha$. The eigenvalues of $A$ are therefore distributed on two circles symmetric to the imaginary axis for any $\alpha \in(0,0.5)$; see Figure 7

Table 2 lists two groups of experimental results. In the first group, we let $\alpha \rightarrow 0.5$ so that the two circles get closer to each other toward the imaginary axis. Both $\Delta(A)$ and dif decrease at the same time. In the second group, we fix $\alpha$ at 0.45 and shift $A$ so that $\Delta(A)$ gets smaller and dif remains the same. Algorithm M-ABQR is more accurate in both cases.

Example 3. The matrix is constructed as

$$
A=Q^{\mathrm{T}}\left(\begin{array}{rr}
A_{11} & A_{12} \\
0 & A_{22}
\end{array}\right) Q
$$

where $Q$ is an orthogonal matrix, $A_{12}$ is a $5 \times 5$ random matrix, $A_{11}$ and $A_{22}$ are random upper triangular matrices except that $\operatorname{diag}\left(A_{11}\right)=-\operatorname{diag}\left(A_{22}\right)=\beta D$ with

TABLE 2. Experimental results with Example $2(k=20)$.

\begin{tabular}{|l|c|c|c|c|c|}
\hline$\Delta(A)$ & dif & iter & $\begin{array}{c}\text { MM-INV } \\
\text { rdr }\end{array}$ & $\begin{array}{c}\text { MM-GQR } \\
\text { rdr }\end{array}$ & $\begin{array}{c}\text { M-ABQR } \\
\text { rdr }\end{array}$ \\
\hline $10^{-1}$ & $1.41 e-1$ & 10 & $6.49 e-16$ & $5.10 e-16$ & $2.77 e-16$ \\
\hline $10^{-3}$ & $1.41 e-3$ & 17 & $6.77 e-14$ & $3.58 e-14$ & $5.32 e-16$ \\
\hline $10^{-5}$ & $1.41 e-5$ & 23 & $5.71 e-11$ & $4.56 e-12$ & $3.28 e-15$ \\
\hline $10^{-7}$ & $1.41 e-7$ & 29 & $6.95 e-08$ & $1.42 e-10$ & $3.64 e-14$ \\
\hline \hline $10^{-3}$ & $1.41 e-1$ & 16 & $2.59 e-15$ & $1.03 e-15$ & $2.90 e-16$ \\
\hline $10^{-5}$ & $1.41 e-1$ & 23 & $2.25 e-14$ & $1.32 e-14$ & $3.27 e-16$ \\
\hline $10^{-7}$ & $1.41 e-1$ & 30 & $1.62 e-13$ & $1.42 e-13$ & $3.00 e-16$ \\
\hline
\end{tabular}


TABLE 3. Experimental results with Example 3.

\begin{tabular}{|c|c|c|c|c|c|c|}
\hline$\beta$ & $\Delta(A)$ & dif & iter & $\begin{array}{c}\text { MM-INV } \\
\text { rdr }\end{array}$ & $\begin{array}{c}\text { MM-GQR } \\
\text { rdr }\end{array}$ & $\begin{array}{c}\text { M-ABQR } \\
\text { rdr }\end{array}$ \\
\hline 1.0 & $2.43 e-1$ & $2.56 e-2$ & 9 & $1.63 e-15$ & $7.42 e-16$ & $4.58 e-16$ \\
\hline 0.5 & $1.21 e-1$ & $2.19 e-3$ & 10 & $1.61 e-14$ & $2.67 e-15$ & $5.08 e-16$ \\
\hline 0.3 & $7.20 e-2$ & $2.33 e-4$ & 11 & $1.34 e-13$ & $3.67 e-14$ & $7.05 e-16$ \\
\hline 0.2 & $4.86 e-2$ & $2.46 e-5$ & 11 & $1.41 e-11$ & $1.31 e-12$ & $4.50 e-15$ \\
\hline 0.1 & $2.43 e-2$ & $1.23 e-7$ & 12 & $6.57 e-10$ & $6.57 e-10$ & $4.83 e-14$ \\
\hline
\end{tabular}

a random positive diagonal matrix and a positive parameter $\beta$. As $\beta$ gets smaller, dif decreases quickly but $\Delta(A)$ remains of the same order. Table 3 shows that, as $\beta$ gets smaller, the accuracy of M-ABQR is much higher than that of MM-INV and MM-GQR.

Our experimental results confirm that the dominant factor for the number of iterations is $\Delta(A)$. The convergence criterion is important in that a premature stop of the iteration would render a poor decoupling residual. Given a converged matrix pair by a one-sided iteration, our scheme for subspace extraction and deflation gives competitive numerical results. We note that our experiments indicate that $\left\|E_{21}\right\|_{\mathrm{F}}$ and $\left\|F_{21}\right\|_{\mathrm{F}}$ computed by the two-sided algorithms are not well balanced.

\section{Concluding REMARKS}

We have related the algebraic structures of the matrix pairs at convergence to that of the initial matrix pairs for the spectral division algorithms based on the matrix sign/disc functions. By exploring such algebraic relationships, we have provided modified spectral division algorithms for the block generalized Schur decomposition (1.1). In the case that only a spectral subspace is required, our deflation scheme can be used, with little extra cost, to obtain an a posteriori estimate on the accuracy of the computed solution. Such an estimate is helpful in that the iteration procedures are not backward stable and a priori error estimates are often overly pessimistic.

Our presentation in this paper is also intended to make spectral division methods more understandable and to reveal their computational advantages and remaining drawbacks. For the spectral division via the matrix sign function, our analysis shows that it is sensitive to perturbations in $B$ as well as in $A$. Preconditioning for the computation of $B A_{k}^{-1} B$ at each step may improve the accuracy, with some tradeoff of arithmetic cost and programming simplicity. For the spectral division via the matrix disc function, our analysis shows that the element decrease in the iterates due to the orthonormal condition of $\left(C_{k}, S_{k}\right)$ entails the preconditioning step for the numerical extraction of a spectral subspace. The effectiveness of our precondition scheme depends on that of rank-revealing algorithms. We have reduced the arithmetic cost of the spectral division methods by nearly $50 \%$; Malyshev's iteration is still rather expensive. We have described a class of Malyshev-like algorithms and presented some necessary convergence conditions. It is an open problem to find more efficient inverse-free algorithms in the class and to design effective acceleration techniques.

In the case in which there are eigenvalues near the spectral dividing curve (the imaginary axis for the matrix sign function or the unit circle for the matrix disc function), the iteration either fails to converge or converges at a nearly linear rate. 
In either case, the iteration is stopped in practice by a prespecified upper bound on the number of iterations.

\section{ACKNOWLEDGMENTS}

We express our gratitude to J. J. Dongarra for bringing our attention to the open problems with spectral division methods and to P. Benner, C. H. Bischof, L. Elsner, D. O'Leary, and G. W. Stewart for their valuable comments and suggestions.

\section{REFERENCES}

[1] W. F. Arnold and A. J. Laub. Generalized eigenproblem algorithms and software for algebraic Riccati equations. Proc. IEEE, 72:1746-1754, 1984.

[2] Z. Bai and J. Demmel. Using the matrix sign function to compute invariant subspaces. SIAM J. Matrix Anal. Appl., 19(1):205-225, 1998. MR 99c:65066

[3] Z. Bai, J. Demmel, and M. Gu. An inverse free parallel spectral divide and conquer algorithm for nonsymmetric eigenproblems. Numer. Math., 76(3):279-308, 1997. MR 98d:65043

[4] Z. Bai, J. W. Demmel, J. Dongarra, A. Petitet, and H. Robinson. The spectral decomposition of nonsymmetric matrices on distributed memory multiprocessors. SIAM J. Sci. Comp., 18:1446-1461, 1997. MR 98d:65027

[5] P. Benner. Contributions to the Numerical Solution of Algebraic Riccati Equations and Related Eigenvalue Problems. Logos-Verlag, Berlin, Germany, 1997.

[6] P. Benner and E. S. Quintana-Ortí. Solving stable generalized Lyapunov equations with the matrix sign function. Numer. Alg., 20(1):75-100, 1999.

[7] C. H. Bischof and G. Quintana-Ortí. Computing rank-revealing QR factorizations of dense matrices. Technical Report MCS-P559-0196, Mathematics and Computer Science Division, Argonne National Laboratory, 1996. ACM Trans. Math. Soft., 24(2): 226-253, 1998.

[8] A. Y. Bulgakov and S. K. Godunov. Circular dichotomy of the spectrum of a matrix. Siberian Math. J., 29(5):734-744, 1988. MR 89m:15005

[9] T. Chan. Rank-revealing QR factorizations. Lin. Alg. Appl., 88/89:67-82, 1987. MR 88c:15011

[10] J. Gardiner and A. J. Laub. A generalization of the matrix sign function solution for algebraic Riccati equations. Int. J. Control, 44:823-832, 1986.

[11] S. K. Godunov. The problem of dichotomy of the spectrum of a matrix. Siberian Math. J., 27(5):649-660, 1986. MR 88d:34066

[12] G. Golub and C. Van Loan. Matrix Computations. The Johns Hopkins University Press, 1989. MR 90d:65055

[13] P. Hong and C. T. Pan. The rank revealing QR and SVD. Math. Comp., 58:213-232, 1992. MR 93d:65031

[14] C. Kenney and A. J. Laub. Rational iterative methods for the matrix sign function. SIAM J. Mat. Anal. Appl., 12:273-291, 1991. MR 92j:15009

[15] V. N. Kublanovskaya. An approach to solving the spectral problem of $A-\lambda B$. In B. Kåagström and A. Ruhe, editors, Matrix Pencils, pages 17-29. Springer-Verlag, New York, 1983. MR 84c:65009

[16] V. N. Kublanovskaya. AB algorithm and its modifications for the spectral problem of linear pencils of matrices. Numer. Math., 43:329-342, 1984. MR 85k:65034

[17] H. Kwakernaak and R. Sivan. Linear Optimal Control Systems. Wiley-Interscience, New York, 1972. MR 53:10394

[18] A. N. Malyshev. Parallel algorithm for solving some spectral problems of linear algebra. Lin. Alg. Appl., 188/189:489-520, 1993. MR 94i:65052

[19] C. Moler and G. W. Stewart. An algorithm for generalized matrix eigenvalue problems. SIAM J. Numer. Anal., 10:241-256, 1973. MR 49:10135

[20] G. Quintana-Ortí and E. S. Quintana-Ortí. Parallel algorithms for computing rank-revealing QR factorizations. In G. Cooperman, G. Micher, and H. Vinck, editors, High Performance Computing and Networking, pages 122-137. Springer-Verlag, Berlin, 1997.

[21] G. Quintana-Ortí, X. Sun, and C. H. Bischof. A BLAS-3 version of the QR factorization with column pivoting. SIAM J. Sci. Comp., 19(5):1486-1494, 1998. 
[22] J. Roberts. Linear model reduction and solution of algebraic Riccati equations by the use of the sign function. Int. J. Control, 32:677-687, 1980. MR 81m:93042

[23] G. W. Stewart. On the sensitivity of the eigenvalue problem. SIAM J. Numer. Anal., 9:669686, 1972. MR 47:244

[24] G. W. Stewart. Perturbation theory for the generalized eigenvalue problem. In G. Golub C. de Boor, editor, Recent Advances in Numerical Analysis, pages 193-206. Academic Press, New York, 1978. MR 80c:65092

[25] G. W. Stewart and J. G. Sun. Matrix Perturbation Theory. Academic Press, New York, 1990. MR 92a:65017

[26] P. Van Dooren. Reducing subspaces: Definitions, properties and algorithms. In B. Kågström and A. Ruhe, editors, Matrix Pencils, pages 17-29. Springer-Verlag, New York, 1983. MR 84c:65009

[27] A. Varga. On stabilization methods of descriptor systems. System Control Let., 24:133-138, 1995. MR 95i:93130

Department of Computer Science, Duke University, D107, Levine Science Research Center, Durham, North Carolina 27708-0129

E-mail address: xiaobai@cs.duke.edu

Departmento de Ingeniería y Ciencia de Computadores, Universidad Jaime I, 12080 Castellón, Spain

E-mail address: quintana@icc.uji.es 